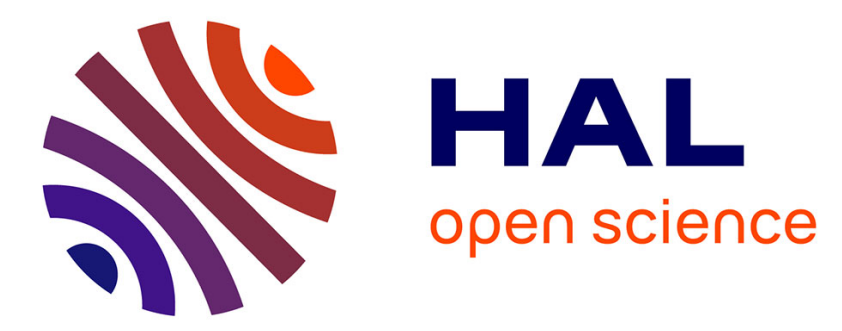

\title{
40 Years of Studying RNA Import into Mitochondria: From Basic Mechanisms to Gene Therapy Strategies P. Kamenski, I. A Krasheninnikov, I. Tarassov
}

\section{To cite this version:}

P. Kamenski, I. A Krasheninnikov, I. Tarassov. 40 Years of Studying RNA Import into Mitochondria: From Basic Mechanisms to Gene Therapy Strategies. Molecular Biology, 2019, 53 (6), pp.924 - 932. 10.1134/S0026893319060074 . hal-02415421

\section{HAL Id: hal-02415421 \\ https://hal.science/hal-02415421}

Submitted on 14 Jan 2020

HAL is a multi-disciplinary open access archive for the deposit and dissemination of scientific research documents, whether they are published or not. The documents may come from teaching and research institutions in France or abroad, or from public or private research centers.
L'archive ouverte pluridisciplinaire HAL, est destinée au dépôt et à la diffusion de documents scientifiques de niveau recherche, publiés ou non, émanant des établissements d'enseignement et de recherche français ou étrangers, des laboratoires publics ou privés. 
40 years of studying RNA import into mitochondria: from basic mechanisms to gene therapy strategies

P.Kamenski $^{1}$, I.A.Krasheninnikov ${ }^{1}$, I.Tarassov $^{2} *$

${ }^{1}$ Department of Molecular Biology, Faculty of Biology, Moscow State University, 1/12 Leninskie Gory, Moscow 119234, Russia

${ }^{2}$ UMR 7154 GMGM, University of Strasbourg - CNRS, 21 rue Rene Descartes, 67084 Strasbourg, France

E-mails for correspondence: peter@protein.bio.msu.ru (P. Kamenski), i.tarassov@unistra@fr(I.Tarassov).

\section{Abstract.}

Mitochondria of many living species internalize nuclear DNA-encoded ribonucleic acids. The pools of imported RNA molecules, as well as fine mechanisms of these processes, are highly species-specific. To date, baker's yeast Saccharomyces cerevisiae are the best studied in this regard. Moreover, the processes of yeast RNA mitochondrial import have been the basis of modeling several gene therapy strategies aimed to palliate negative effects of pathogenic mutations in human mitochondrial DNA. In this review, we summarize our current knowledge about the molecular events taking place in course of yeast RNA import into mitochondria. Also, we describe how this process can be used for compensation of pathogenic mutations in mitochondrial genomes of humans.

Key words

mitochondria, non-coding RNAs, RNA import, targeting, mitochondrial translation, mitochondrial diseases, gene therapy, yeast, human 


\section{MOLECULAR MECHANISMS OF RNA IMPORT INTO MITOCHONDRIA IN YEAST MODEL}

Since their discovery in XIX century, mitochondria were constantly raising the attention of researchers. Nowadays, an endosymbiotic theory of mitochondria origin is generally accepted (for review, see [1]). This theory says that a far ancestor of mitochondria was a free-living bacteria related to modern Rickettsia. In course of evolution, mitochondrial genes migrated to nucleus by currently unknown mechanism. However, modern mitochondria still contain DNA with several proteins and RNAs being encoded therein. On the other hand, these macromolecules are not enough for maintenance of mitochondrial functions. Thus, most of organellar proteins and some RNAs are encoded by nuclear genome and imported into mitochondria from the cytosol.

First data on protein import into mitochondria were obtained in the late 1970s [2]. This area of science has been quickly and constantly developed, and at present, the complex system of protein transport across mitochondrial membranes is described in great detail [3].

The phenomenon of RNA import into mitochondria was first hypothesized in some protozoans [4] and then found in yeast by Robert Martin et al. at Strasbourg University, France [5]. This team analyzed yeast mitochondrial tRNAs by 2D gel-electrophoresis and was able to detect one single species of nucleus-encoded tRNA, namely lysine tRNA with CUU anticodon (further referred to as tRK1). It has to be mentioned that the major tRK1 pool is localized in the cytosol where this tRNA takes part in translation; only 2-5\% of tRK1 molecules were found in mitochondria [5]. For many years, this work remained single one in this field. One of the possible reasons for that might be some distrust of the scientific community in these results. Many researchers simply did not believe in import of negativelycharged RNA molecule across negatively-charged mitochondrial membranes. Furthermore, the exact function of this imported tRNA species remained obscure. Nowadays, however, it is widely accepted that many RNA species are imported to mitochondria of different organisms $[6,7]$.

Baker's yeast has become a first model organism for mechanistic studies of RNA import into mitochondria. In the late 1980s, Ivan Tarassov and Nina Entelis (Department of Molecular Biology, Faculty of Biology, Moscow State University, Russia), developed the in vivo and in vitro systems to study RNA mitochondrial transport [8]. In vivo system required the electroporation of yeast cells with a radioactively-labelled tRNA and further radioautographic detection of this tRNA in the mitochondrial RNA fraction. Indeed, tRK1 was detected both in the cytosol and in mitochondria while all other cytosolic tRNAs, when 
delivered into the yeast cells, were localized exclusively in cytosol. In vitro approach involved incubation of isolated yeast mitochondria with radioactively-labeled tRNA. By this approach, it was confirmed that, among all yeast tRNAs, only tRK1 is able to cross mitochondrial membranes. This process was found to be ATP-dependent and to require some unidentified, at this stage, soluble yeast cytosolic proteins [8].

Further studies of tRK1 import into yeast mitochondria were conducted jointly in the two teams, at Moscow and Strasbourg Universities. The main question was about the exact molecular mechanisms of tRNA translocation across mitochondrial membranes. It was shown that tRK1 import may be related to protein mitochondrial import machinery: several components of protein import apparatus were proven to take part in tRK1 transport [9]. Among them, there were Tom20p (imported proteins receptor in the outer membrane) and Tim $44 p$ (component of the so-called "protein import motor" in the inner membrane). Another finding was the dependence of tRK1 import on the mitochondrial membrane electrochemical potential $\Delta \Psi$ [9]. Taken together, these results have let to hypothesize that tRK1 uses protein import pathway to cross the mitochondrial membranes. Later studies, however, provided some evidence that a channel for tRNA import in the outer membrane might be not only Tom40p (the only channel for protein transport) but also voltage-dependent anion channel (VDAC) [10]. The same situation when both Tom40 and VDAC are involved in tRNA mitochondrial transport was described in higher plants [11]. Nevertheless, the receptor components of protein import machinery seem to assure tRNA delivery into mitochondria [12].

It is worth mentioning that there are two lysine tRNAs encoded in yeast nuclear genome, and the second one (further referred to as tRK2), with UUU anticodon, differs from tRK1 by 21 bases. In contrast to tRK1, tRK2 has never been detected in mitochondria. This means that there should exist determinants that ensure the import for tRK1 and/or import antideterminants for tRK2. Most obvious type of these determinants would be nucleotides that differ between tRK1 and tRK2. Applying in vitro and in vivo tRNA mitochondrial import systems, this hypothesis was verified by creation of sets of mutant tRK1 and tRK2 versions where differing bases were one-by-one substituted by the corresponding bases of tRK2 and tRK1, respectively. These mutant tRNAs were assayed for their import ability; it was found that, indeed, several tRK1 nucleotides serve as its import determinants, namely the first anticodon nucleotide (C34) [13], first base pair of the acceptor stem (G1-U72), and discriminator nucleotide U73 [14].

These nucleotides could ensure the interaction of tRK1 with putative protein factors of the import process [8]. Further studies permitted to identify several of them.

First protein factor participating in tRK1 mitochondrial targeting was identified as 
the cytosolic lysyl-tRNA synthetase (Krs1p) [15]. Normally, for the needs of cytosolic translation, it aminoacylates tRK1 in the cytosol. So far, it was demonstrated that this aminoacylation also makes this tRNA import-competent. However, the aminoacylated state itself is not a prerequisite for tRK1 to be imported. Several mutant versions of tRK1 have been created that could not be aminoacylated by whatever amino acid but still have been imported into mitochondria in both in vitro and in vivo systems $[13,14,16]$. It has been then hypothesized that lysinylation of tRK1 might be important for some further steps of the import process.

This last hypothesis has been experimentally proven when other protein factor implicated in tRK1 import has been identified. This factor was the cytosolic precursor of mitochondrial lysyl-tRNA synthetase (preMsk1p) which is synthesized by cytosolic ribosomes and then imported into mitochondria where, in its mature form, it aminiocylates mitochondrially-encoded lysine tRNA (further referred to as tRK3). It has been demonstrated that in yeast strain devoid of MSK1 gene no tRK1 is detected in mitochondria [15]. In vitro tRK1, if aminoacylated, binds preMsk1p which is necessary for tRNA transport across the membranes of isolated mitochondria, while preMsk1p can neither bind deacylated tRK1 nor aminoacylate it [15]. Interestingly, tRNA import-driving properties of recombinant preMsk1p in vitro depend on the mode of its purification: preMsk1p purified from E.coli under denaturating conditions with subsequent renaturation step, can bind tRK1 and direct its import while natively-purified protein is not competent in this process [17]. Using different truncated versions of preMsk1p, it has been shown that its tRK1-binding and import-directing activity is localized in the N-terminal domain $[18,19]$ which, when protein functions as aminoacyl-tRNA synthetase, is responsible for primary tRNA anticodon recognition.

Regarding tRK1 and tRK2 versions mentioned earlier, there is solid correlation between their abilities to bind preMsk1p and efficiencies of their import into mitochondria $[13,14,16]$. Furthermore, mutant tRNAs that could not be aminoacylated but were successfully imported also demonstrated strong affinity to preMsk1p. This finding may be explained if tRK1 aminoacylation is essentially needed to permit formation of complex with preMsk1p. Concerning functional significance of this complex, it is assumed that tRK1 might be imported into mitochondrial being bound to preMsk1p. In other words, this protein could be a carrier for tRNA translocation through mitochondrial membranes.

The third identified protein factor of tRK1 import into yeast mitochondria is second isoform of glycolytic enzyme enolase (Eno2p) [20]. Together with preMsk1p, Eno2p can drive aminoacylated tRK1 into isolated yeast mitochondria. This protein was shown to bind tRK1 (if it is aminoacylated) and to facilitate the formation of complex between this tRNA 
and preMsk1p [20]. Moreover, in in vitro experiments it was demonstrated that Eno2p is partially localized to the surface of mitochondria [20,21]. At the same time, preMsk1p is synthesized, at least partially, by ribosomes associated with the outer mitochondrial membranes [20]. Taken together, these data are in favor of the targeting function of Eno2p. This protein could bind tRK1 in the cytosol and then deliver it to the mitochondrial surface where tRK1 is released by Eno2p to bind nascent preMsk1p. The next step might be translocation of the latter complex through mitochondrial membranes.

Finalizing the description of protein factors of tRK1 import, it has to be mentioned that the list of these factors is definitely not completed. In a more recent study, it has been shown that additional yeast proteins might be involved in this process [22].

Another very important aspect of tRNA import into mitochondria concerns structural requirements of the RNA molecule. First indication on the significance of tRNA structure for its import were detected in the experiments where "nicked" tRK1 (RNA reconstituted from two moieties and thus containing a single-stranded break) were able to reach mitochondrial matrix but only if the two tRNA halves were pre-annealed [13]. The ability of tRK1 to fold into the proper ternary structure is thus a prerequisite for its import. Later, using in-gel FRET technique, it was shown that tRK1 can be folded into two different conformations: one is the classical tRNA L-shape, while second is characterized by alternative base-pairing between 3' part of AA-stem and the T-loop [23]. This latter structure could be detected when tRK1 was bound to Eno2p. This finding thus provided a link in the chain of events leading to tRK1 import into mitochondria: Eno2p can function as RNA-chaperone which induces structural changes in the tRK1 molecule upon interaction with the protein, and this structural rearrangement defines the ability of tRK1 to be imported. In silico modeling showed that no other yeast cytosolic tRNA could fold into such alternative structure [23]. This model explains why only tRK1 is imported into mitochondria: its nucleotide sequence allows its existence in the alternative form which is fixed by Eno2p. This, in turn, ensures tRK1 further interaction with preMsk1p and subsequent translocation across mitochondrial membranes. The chain of events in the cytosol preceding tRK1 import through organellar membranes is schematically represented in Figure 1.

According to in silico predictions, alternative structure of tRK1 is characterized by formation of two hairpins, "F" and "D" [23]. It has been proposed that F-hairpin as structural element is enough to ensure RNA transport into mitochondria. Indeed, analyzing a set of small artificial RNAs, it has been shown that RNA molecules with stable F-hairpin are imported into yeast mitochondria both in vitro and in vivo with high efficiencies. The maximal efficiencies of import were documented for the RNA versions containing F- and D-hairpins 
together [23].

All data and conclusions mentioned above are related to the question "how tRK1 is imported into yeast mitochondria". Another important and maybe even more intriguing question is "WHY it is imported". For a long time, the answer to this question was not evident. Apparently, it is logical to assume that tRK1 takes part in mitochondrial translation upon its import. Indeed, such participation has been demonstrated: imported tRNA derivatives are able to suppress nonsense mutations in yeast mitochondrial DNA [24]. However, physiological role of tRK1 import remained unclear. Mitochondrial genome of yeast codes for lysine tRNA (tRK3) with 5-carboxymethylaminomethyl-2-thioUUU anticodon which is able to decode both lysine codons, AAA and AAG [25]. Thus, participation of tRK1 in mitochondrial translation, albeit possible, seemed to be dispensable. Best approach to clarify this issue would be to switch off tRK1 import and to look for the effects on mitochondrial function. This has been done by exchanging MSK1 gene from Saccharomyces cerevisiae genome by the orthologous gene from another yeast, Ashbya gossypii. The precursor of mitochondrial lysyl-tRNA synthetase from this organism was completely incompetent in driving tRK1 import into mitochondria of S.cerevisiae but was imported into mitochondria and aminoacylated tRK3 [18]. It has been shown that mitochondrial function (OXPHOS system activity, characterized by yeast growth on special "respiration" media) of the recombinant strain was not altered at normal growth conditions but was significantly decreased at elevated temperatures $\left(37^{\circ} \mathrm{C}\right)$. Surprisingly, this was due to tRK3: at $37^{\circ} \mathrm{C}$, the wobble U34 of this tRNA becomes hypo-modified which leads to inefficient recognition of AAG codons in mitochondrial mRNAs. In this situation, tRK1, which bears the non-modified CUU anticodon, becomes obligatory for mitochondrial translation [18]. Thus, tRK1 import seems to be related to adaptation mechanism of mitochondrial translation to elevated temperatures.

\section{YEAST RNAS IMPORT INTO HUMAN MITOCHONDRIA AS A WAY TO ADDRESS THE PATHOGENIC MUTATIONS}

As it has already been mentioned above, several derivatives of yeast tRK1 are able to suppress mutations in yeast mitochondrial DNA [24]. Nowadays, more that 200 pathogenic mutations in human mitochondrial DNA have been described (for review, see [26]). Being among most common ones, mutations in mitochondrial tRNA genes lead to abnormal translation in organelles and to significant loss of mitochondrial functionality. tRNA import 
from the cytosol might become a good tool for suppression of these mutations. The relevance of natural tRNA mitochondrial import into human mitochondria is still questionable. There has been a single report suggesting the import of two glutamine tRNA isoacceptor species. Authors have hypothesized that these tRNAs are imported in order to alleviate the absence of the transamidation pathway in human mitochondria which is necessary to generate these tRNAs [27]. So far a similar hypothesis for the yeast system [28] was contradicted by further studies [29]. On the other hand, taking into account similarities of transport machineries between yeast and mammalians, it was tentative to see if such a targeting could be established artificially. Thus, it was worth trying to direct yeast tRNAs into human mitochondria in hope that they would be able to take part in organellar translation. This could be a way to address negative effects of pathogenic mutations. Indeed, it has been demonstrated that yeast tRK1 and its mutant derivatives have been imported into isolated human mitochondria [24]. It has also been shown that all the requirements of tRK1 import into isolated human mitochondria are similar to those described for yeast ones [30]. Moreover, human orthologues of preMsk1p [31] and Eno2p [32] were demonstrated to take part in this artificially established targeting. Thus, the cryptic machinery of tRNA import seems to be present in human. Moreover, abovementioned small artificial RNAs with stable F- and/or D-hairpins were also imported into isolated human mitochondria with efficiencies being even several times higher than that of a full-sized tRK1 [23].

Once positive results were obtained in in vitro system, yeast tRK1 derivatives were then tested for their ability to cross the mitochondrial membranes in cultured human cells. Indeed, tRNA import was detected in vivo [33]. Thus, it seemed possible to use tRNA import pathway to address a patogenic mutation in human mitochondrial DNA. One of the most frequent human mtDNA mutations, A8344G, was previously characterized in the lysine tRNA gene. This mutation causes co-called MERRF syndrome (myoclonic epilepsy with ragged-red fibers) which is linked to the defects of tRNA aminoacylation and premature termination of mitochondrial translation on the lysine codons [34]. Yeast tRK1 or tRK2 importable derivatives, when introduced into cultured human cells harboring MERRF mutation, partially restored pathological phenotype due to improvement of mitochondrial translation [33]. This study was the very first case of alleviation of mutation in the mitochondrial tRNA gene by an imported RNA. Later, to broaden the spectrum of mutations in mitochondrial DNA that might be addressed via tRNA import, another yeast tRK1 derivative was constructed which kept all the determinants of its mitochondrial import but the determinants of aminoacylation with lisyne were exchanged to provide the tRNA leucine identity [35]. This permitted to address another common mutation, A3243G, which is localized in the mitochondrial leucine tRNA 
gene and causes the MELAS syndrome (mitochondrial encephalomyopathy, lactic acidosis, and stroke-like episodes). This mutation, similarly to MERRF one, leads to the abnormal mitochondrial translation, through alteration of anticodon modification [36,37]. Abovedescribed artificial tRNA could potentially be aminoacylated with leucine in human mitochondria and thus might take part in mitochondrial translation instead of mutated leucine tRNA in MELAS mutation bearing cells [35]. Thus, two point mutations in two different mitochondrial tRNA genes were successfully addressed by exploiting RNA mitochondrial import pathway.

Another relatively frequent mutation types in human mitochondrial DNA are large deletions. These commonly lead to simultaneous deletion of several genes from mtDNA. Human cells with such deletions are usually viable, due to the heteroplasmy phenomenon, common for a vast majority of human mtDNA mutations. Heteroplasmy is the simultaneous presence of mutant and wild-type mtDNA in the same cell. For many pathogenic mitochondrial mutations, there are threshold levels of heteroplasmy: if the proportion of mutant molecules is below this level, there is no mutant phenotype. Above this threshold (depending on the mutation between 60 and 80\%), the severity of symptoms depends on the heteroplasmy level. Correspondingly, decreasing the heteroplasmy level might be one of the strategies to address mtDNA mutations.

The shorter versions of importable RNAs (containing F- and D-hairpins) were then used as vectors to target mutant mtDNA harboring a large deletion associated with the KSS (Kearns-Sayre syndrome). The KSS deletion creates a unique sequence which served as a target. Short oligonucleotides complementary to one or another mtDNA strand containing this unique sequence was either inserted between F- and D- import determinants or simply fused to one of them. It was demonstrated that such "anti-replicative" RNA molecules are partially targeted into mitochondrial matrix and affect, in a specific way, progression of the replication fork of mutant mtDNA, which resulted in a significant, although transient, decrease of proportion of KSS deletion-bearing DNA molecules [38]. Later, the same strategy has been shown to effectively decrease heteroplasmy level even in case of point mutations in mitochondrial DNA, with the model of a mutation in the $5^{\text {th }}$ subunit of NADH-dehydrogenase [39].

\section{PERSPECTIVES}

Much is known nowadays about basic mechanisms of tRNA import into yeast and human mitochondria. However, there are several topics to be further studied. First of all, the 
process of tRK1 translocation across mitochondrial membranes needs to be investigated. As it has been described above, protein import machinery and voltage-dependent anion channels seem to be involved in tRK1 transport through the yeast mitochondrial outer membrane. It is not clear whether discrimination between these two channels takes place or tRK1 has no preferences in choosing the exact pathway of outer membrane translocation. Also, nothing is known about how tRK1 crosses the inner membrane and reaches mitochondrial matrix. Modern microscopy methods and cross-linking experiments could help to resolve these issues and to robustly identify proteins that take part in tRK1 translocation as membrane channels and as receptors/regulators of this process.

Another intriguing issue is the regulation of tRK1 import into mitochondria. Only 3$5 \%$ of tRK1 cytosolic pool is targeted into mitochondria, while the rest molecules are involved in translation in cytoplasm. How the selection of tRK1 molecules takes place is not clear. It is rather possible that Eno2p interaction is responsible for that, but some other factors might also take part in targeting [22]. On the other hand, the proportion of imported tRK1 molecules could be also subjected to regulation: it is known that ubiquitin-proteasome system has something to do with such regulation [40]. Of no doubt is that regulation of tRK1 import should be comprehensively studied in future.

Investigations aimed to the utilization of tRNA import as gene therapy tool are quite complex and technically complicated. Nevertheless, as it has been described before, great progress has been made in this field during past 15-20 years. Several strategies for suppression of pathogenic mutations in human mitochondrial DNA based on RNA import have been developed. However, they all share some common disadvantages that significantly decrease their medical potential. Among most important disadvantages, there are low efficiency and transient mode of action of the discussed approaches. These difficulties were partially overcome. It has been shown that introduction of desoxyribonucleotides in the sequence of "therapeutic" RNAs and chemical modifications of their ribonucleotides increase the lifespan of these molecules [41]. Also, a method of carrier-free RNA delivery into mitochondria was established based on the RNA cleavable conjugation with cholesterol [42]. These approaches are potentially important for increasing the efficiency of therapeutic effect of RNA import pathway. In any case, studies should be continued in this direction.

There is a very important issue which should be addressed using RNA mitochondrial import, namely targeting CRISPR/Cas9 system into mitochondria. This genome editing technology is robustly recognized as the most powerful tool for making directed changes in the genomic DNA of any living creature. Human mitochondrial DNA can be edited by Znfinger recombinant nucleases $[43,44,45]$ or TALEN approach [46]. In these technologies, no 
RNA components are used, and there are only proteins to be imported into mitochondria. In case of CRISPR/Cas9, both protein and RNA components should be targeted into the organelles. Moderate progress has been achieved recently on this way. It was demonstrated that guide RNAs with introduced above-described import determinants are imported into mitochondria of human cells, as well as Cas9 protein supplemented with the mitochondrial targeting sequence [47]. Using CRISPR/Cas9 in mitochondria might be somewhat more complex if compared with the traditional nuclear DNA-targeted technology, but nevertheless, the possibility of this system to be functionally active in human mitochondria has been experimentally shown [47]. In a very recent work, an exogenous DNA sequence has been inserted into zebrafish mitochondrial DNA using CRISPR/Cas9 approach, and moreover, this DNA has been efficiently transmitted from $F_{0}$ to $F_{1}$ generation [48]. Taken together, these data allow being in hopes that efficient version of mitochondrially-addressed CRISPR/Cas9 system may be developed in future.

\section{ACKNOWLEDGMENTS}

We thank all past and present members of Strasbourg and Moscow labs who have obtained most of the above-described data. Our special thanks are to Ivan Chicherin (Moscow University) who has prepared the illustration for this review. The authors are grateful to French National Research Center (CNRS) and Russian Foundation for Basic Research (RFBR) that constantly supported their joint studies. For the moment, these are supported by RFBR grant № 17-54-16005. IT is supported by the LabEx (Laboratory of Excellence grant) MitoCross, LIA (Laboratoire Internationale Associé) ARN-mitocure and the EUR IMCBio grant.

\section{FIGURE LEGEND}

Events that ensure tRK1 translocation across yeast mitochondrial membranes.

\section{REFERENCES}

1. Martin, W. F., Garg, S., Zimorski, V. (2015) Endosymbiotic theories for eukaryote origin, Philos Trans R Soc Lond B Biol Sci. 370, 20140330.

2. Hallermayer, G., Zimmermann, R., Neupert, W. (1977) Kinetic studies on the transport of cytoplasmically synthesized proteins into the mitochondria in intact cells of 
Neurospora crassa, Eur J Biochem. 81, 523-532.

3. Pfanner, N., Warscheid, B., Wiedemann, N. (2019) Mitochondrial proteins: from biogenesis to functional networks, Nat Rev Mol Cell Biol. 20, 267-284.

4. Suyama, Y. (1967) The origins of mitochondrial ribonucleic acids in Tetrahymena pyriformis, Biochemistry. 6, 2829-2839.

5. Martin, R. P., Schneller, J. M., Stahl, A. J., Dirheimer, G. (1979) Import of nuclear deoxyribonucleic acid coded lysine-accepting transfer ribonucleic acid (anticodon CU-U) into yeast mitochondria, Biochemistry. 18, 4600-4605.

6. Verechshagina, N. A., Konstantinov, Y. M., Kamenski, P. A., Mazunin, I. O. (2018) Import of Proteins and Nucleic Acids into Mitochondria, Biochemistry (Mosc). 83, 643-661.

7. Jeandard, D., Smirnova, A., Tarassov, I., Barrey, E., Smirnov, A., Entelis, N. (2019) Import of Non-Coding RNAs into Human Mitochondria: A Critical Review and Emerging Approaches, Cells. 8.

8. Tarassov, I. A., Entelis, N. S. (1992) Mitochondrially-imported cytoplasmic tRNA(Lys)(CUU) of Saccharomyces cerevisiae: in vivo and in vitro targetting systems, Nucleic Acids Res. 20, 1277-1281.

9. Tarassov, I., Entelis, N., Martin, R. P. (1995) An intact protein translocating machinery is required for mitochondrial import of a yeast cytoplasmic tRNA, J Mol Biol. 245, 315-323.

10. Vyssokikh, M. Y., Schirtz, T., Kolesnikova, O., Entelis, N., Antonenko, Y. N., Rokitskaya, T. I., Tarassov, I. (2012) Isoform porin 2 is involved in tRNA(Lys) transport from cytosol to mitochondria in yeast, BBA Bioenergetics. 1817, S124-S125.

11. Salinas, T., Duchene, A. M., Delage, L., Nilsson, S., Glaser, E., Zaepfel, M., Marechal-Drouard, L. (2006) The voltage-dependent anion channel, a major component of the tRNA import machinery in plant mitochondria, Proc Natl Acad Sci U S A. 103, 18362-18367.

12. Schirtz, T. (2012) Etude du mécanisme de translocation de l'ARNtLys(CUU) dans les mitochondries de Saccharomyces cerevisiae, PhD Thesis, Strasbourg University, Strasbourg.

13. Entelis, N., Kieffer, S., Kolesnikova, O., Martin, R., Tarassov, I. (1998) Structural requirement of tRNA ${ }^{\text {Lys }}$ for its import into yeast mitochondria, Proc. Natl. Acad. Sci. USA. 95, 2838-2843.

14. Kazakova, H. A., Entelis, N. S., Martin, R. P., Tarassov, I. A. (1999) The aminoacceptor stem of the yeast tRNA(Lys) contains determinants of mitochondrial import selectivity, FEBS Lett. 442, 193-197. 
15. Tarassov, I., Entelis, N., Martin, R. P. (1995) Mitochondrial import of a cytoplasmic lysine-tRNA in yeast is mediated by cooperation of cytoplasmic and mitochondrial lysyl-tRNA synthetases, Embo J. 14, 3461-3471.

16. Kolesnikova, O., Entelis, N., Kazakova, H., Brandina, I., Martin, R. P., Tarassov, I. (2002) Targeting of tRNA into yeast and human mitochondria: the role of anticodon nucleotides, Mitochondrion. 2, 95-107.

17. Smirnova, E. V., Chicherin, I. V., Baleva, M. V., Entelis, N. S., Tarassov, I. A., Kamenski, P. A. (2016) Procedure for Purification of Recombinant preMsk1p from E. coli Determines Its Properties as a Factor of tRNA Import into Yeast Mitochondria, Biochemistry (Mosc). 81, 1081-1088.

18. Kamenski, P., Kolesnikova, O., Jubenot, V., Entelis, N., Krasheninnikov, I. A., Martin, R. P., Tarassov, I. (2007) Evidence for an adaptation mechanism of mitochondrial translation via tRNA import from the cytosol, Mol Cell. 26, 625-637.

19. Kamenski, P., Smirnova, E., Kolesnikova, O., Krasheninnikov, I. A., Martin, R. P., Entelis, N., Tarassov, I. (2010) tRNA mitochondrial import in yeast: Mapping of the import determinants in the carrier protein, the precursor of mitochondrial lysyl-tRNA synthetase, Mitochondrion. 10, 284-293.

20. Entelis, N., Brandina, I., Kamenski, P., Krasheninnikov, I. A., Martin, R. P., Tarassov, I. (2006) A glycolytic enzyme, enolase, is recruited as a cofactor of tRNA targeting toward mitochondria in Saccharomyces cerevisiae, Genes Dev. 20, 1609-1620.

21. Brandina, I., Graham, J., Lemaitre-Guillier, C., Entelis, N., Krasheninnikov, I., Sweetlove, L., Tarassov, I., Martin, R. P. (2006) Enolase takes part in a macromolecular complex associated to mitochondria in yeast, Biochim Biophys Acta. 1757, 1217-1228.

22. Baleva, M. V., Meyer, M., Entelis, N., Tarassov, I., Kamenski, P., Masquida, B. (2017) Factors beyond Enolase 2 and Mitochondrial Lysyl-tRNA Synthetase Precursor Are Required for tRNA Import into Yeast Mitochondria, Biochemistry (Mosc). 82, 1324-1335.

23. Kolesnikova, O., Kazakova, H., Comte, C., Steinberg, S., Kamenski, P., Martin, R. P., Tarassov, I., Entelis, N. (2010) Selection of RNA aptamers imported into yeast and human mitochondria, RNA. 16, 926-941.

24. Kolesnikova, O. A., Entelis, N. S., Mireau, H., Fox, T. D., Martin, R. P., Tarassov, I. A. (2000) Suppression of mutations in mitochondrial DNA by tRNAs imported from the cytoplasm, Science. 289, 1931-1933.

25. Umeda, N., Suzuki, T., Yukawa, M., Ohya, Y., Shindo, H., Watanabe, K. (2005) Mitochondria-specific RNA-modifying Enzymes responsible for the biosynthesis of the wobble base in mitochondrial tRNAs. Implications for the molecular pathogenesis of human 
mitochondrial diseases, J Biol Chem. 280, 1613-1624.

26. Patrushev, M. V., Kamenski, P. A., Mazunin, I. O. (2014) Mutations in mitochondrial DNA and approaches for their correction, Biochemistry (Mosc). 79, 1151-1160.

27. Rubio, M. A., Rinehart, J. J., Krett, B., Duvezin-Caubet, S., Reichert, A. S., Soll, D., Alfonzo, J. D. (2008) Mammalian mitochondria have the innate ability to import tRNAs by a mechanism distinct from protein import, Proc Natl Acad Sci U S A. 105, 91869191.

28. Rinehart, J., Krett, B., Rubio, M. A., Alfonzo, J. D., Soll, D. (2005) Saccharomyces cerevisiae imports the cytosolic pathway for Gln-tRNA synthesis into the mitochondrion, Genes Dev. 19, 583-592.

29. Frechin, M., Senger, B., Braye, M., Kern, D., Martin, R. P., Becker, H. D. (2009) Yeast mitochondrial Gln-tRNA(Gln) is generated by a GatFAB-mediated transamidation pathway involving Arc1p-controlled subcellular sorting of cytosolic GluRS, Genes Dev. 23, 1119-1130.

30. Entelis, N. S., Kolesnikova, O. A., Dogan, S., Martin, R. P., Tarassov, I. A. (2001) 5 S rRNA and tRNA Import into Human Mitochondria. Comparison of in vitro requirements., J Biol Chem. 276, 45642-45653.

31. Gowher, A., Smirnov, A., Tarassov, I., Entelis, N. (2013) Induced tRNA import into human mitochondria: implication of a host aminoacyl-tRNA-synthetase, PLoS One. 8, e66228.

32. Baleva, M., Gowher, A., Kamenski, P., Tarassov, I., Entelis, N., Masquida, B. (2015) A Moonlighting Human Protein Is Involved in Mitochondrial Import of tRNA, Int J Mol Sci. 16, 9354-9367.

33. Kolesnikova, O. A., Entelis, N. S., Jacquin-Becker, C., Goltzene, F., Chrzanowska-Lightowlers, Z. M., Lightowlers, R. N., Martin, R. P., Tarassov, I. (2004) Nuclear DNA-encoded tRNAs targeted into mitochondria can rescue a mitochondrial DNA mutation associated with the MERRF syndrome in cultured human cells, Hum Mol Genet. 13, 2519-2534.

34. Enriquez, J. A., Chomyn, A., Attardi, G. (1995) MtDNA mutation in MERRF syndrome causes defective aminoacylation of tRNA(Lys) and premature translation termination, Nat Genet. 10, 47-55.

35. Karicheva, O. Z., Kolesnikova, O. A., Schirtz, T., Vysokikh, M. Y., MagerHeckel, A. M., Lombes, A., Boucheham, A., Krasheninnikov, I. A., Martin, R. P., Entelis, N., Tarassov, I. (2011) Correction of the consequences of mitochondrial 3243A $>\mathrm{G}$ mutation in the MT-TL1 gene causing the MELAS syndrome by tRNA import into mitochondria, Nucleic 
Acids Res. 39, 8173-8186.

36. Kirino, Y., Goto, Y., Campos, Y., Arenas, J., Suzuki, T. (2005) Specific correlation between the wobble modification deficiency in mutant tRNAs and the clinical features of a human mitochondrial disease, Proc Natl Acad Sci U S A. 102, 7127-7132.

37. Kirino, Y., Yasukawa, T., Ohta, S., Akira, S., Ishihara, K., Watanabe, K., Suzuki, T. (2004) Codon-specific translational defect caused by a wobble modification deficiency in mutant tRNA from a human mitochondrial disease, Proc Natl Acad Sci U S A. 101, 15070-15075.

38. Comte, C., Tonin, Y., Heckel-Mager, A. M., Boucheham, A., Smirnov, A., Aure, K., Lombes, A., Martin, R. P., Entelis, N., Tarassov, I. (2013) Mitochondrial targeting of recombinant RNAs modulates the level of a heteroplasmic mutation in human mitochondrial DNA associated with Kearns Sayre Syndrome, Nucleic Acids Res. 41, 418-433.

39. Tonin, Y., Heckel, A. M., Vysokikh, M., Dovydenko, I., Meschaninova, M., Rotig, A., Munnich, A., Venyaminova, A., Tarassov, I., Entelis, N. (2014) Modeling of antigenomic therapy of mitochondrial diseases by mitochondrially addressed RNA targeting a pathogenic point mutation in mitochondrial DNA, J Biol Chem. 289, 13323-13334.

40. Brandina, I., Smirnov, A., Kolesnikova, O., Entelis, N., Krasheninnikov, I. A., Martin, R. P., Tarassov, I. (2007) tRNA import into yeast mitochondria is regulated by the ubiquitin-proteasome system, FEBS Lett. 581, 4248-4254.

41. Tonin, Y., Heckel, A. M., Dovydenko, I., Meschaninova, M., Comte, C., Venyaminova, A., Pyshnyi, D., Tarassov, I., Entelis, N. (2014) Characterization of chemically modified oligonucleotides targeting a pathogenic mutation in human mitochondrial DNA, Biochimie. 100, 192-199.

42. Dovydenko, I., Tarassov, I., Venyaminova, A., Entelis, N. (2016) Method of carrier-free delivery of therapeutic RNA importable into human mitochondria: Lipophilic conjugates with cleavable bonds, Biomaterials. 76, 408-417.

43. Minczuk, M., Papworth, M. A., Miller, J. C., Murphy, M. P., Klug, A. (2008) Development of a single-chain, quasi-dimeric zinc-finger nuclease for the selective degradation of mutated human mitochondrial DNA, Nucleic Acids Res. 36, 3926-3938.

44. Gammage, P. A., Rorbach, J., Vincent, A. I., Rebar, E. J., Minczuk, M. (2014) Mitochondrially targeted ZFNs for selective degradation of pathogenic mitochondrial genomes bearing large-scale deletions or point mutations, EMBO Mol Med. 6, 458-466.

45. Gammage, P. A., Gaude, E., Van Haute, L., Rebelo-Guiomar, P., Jackson, C. B., Rorbach, J., Pekalski, M. L., Robinson, A. J., Charpentier, M., Concordet, J. P., Frezza, C., Minczuk, M. (2016) Near-complete elimination of mutant mtDNA by iterative or dynamic 
dose-controlled treatment with mtZFNs, Nucleic Acids Res. 44, 7804-7816.

46. Bacman, S. R., Williams, S. L., Pinto, M., Peralta, S., Moraes, C. T. (2013) Specific elimination of mutant mitochondrial genomes in patient-derived cells by mitoTALENs, Nat Med. 19, 1111-1113.

47. Loutre, R., Heckel, A. M., Smirnova, A., Entelis, N., Tarassov, I. (2018) Can Mitochondrial DNA be CRISPRized: Pro and Contra, IUBMB Life. 70, 1233-1239.

48. Bian, W. P., Chen, Y. L., Luo, J. J., Wang, C., Xie, S. L., Pei, D. S. (2019) Knock-In Strategy for Editing Human and Zebrafish Mitochondrial DNA Using MitoCRISPR/Cas9 System, ACS Synth Biol. 8, 621-632.

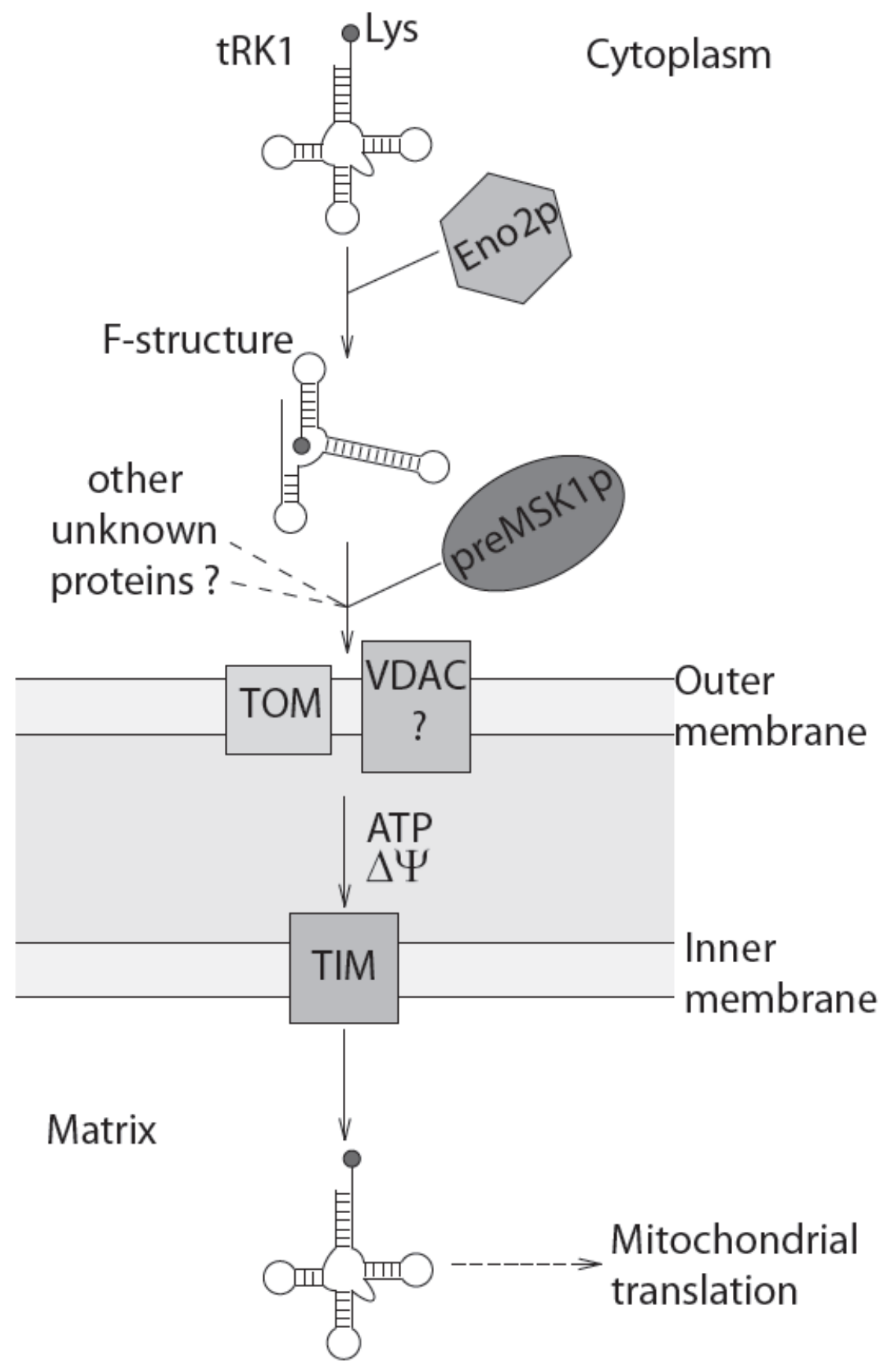

